\title{
LA CONSOLIDACIÓN DE LA PLANIFICACIÓN TERRITORIAL PARA EL DESARROLLO Una mirada a través de la actividad de los centros de formación del Cono Sur
}

\author{
Monti, Alejandra \\ Universidad: Pontificia Universidad Católica de Chile \\ Director de la investigación: Dr. arq. Horacio Torrent \\ E-Mail: montialejandra@gmail.com
}

\section{Resumen}

En el marco de las transformaciones del mapa geopolítico en los años posteriores a la Segunda Guerra Mundial, América Latina adquiere un renovado interés de parte de sus vecinos continentales. Estos vínculos se articulan desde tres áreas de las relaciones internacionales: la cooperación económica, técnica y política, que van a marcar el pulso de las transformaciones en la región con el fin de dar respuestas a la condición de "atraso".

Entendiendo a Latinoamérica como "el" espacio de la planificación para el desarrollo, proponemos una lectura que permita avanzar en las formas y mecanismos de consolidación de la disciplina a escala regional y sus re-interpretaciones en sede local, tomando como objeto de estudio la actividad de dos centros relevantes: el Centro de Estudios Urbanos y Regionales (CEUR) y el Centro Interdisciplinario de Desarrollo Urbano y Regional (CIDU-Chile)

Palabras Clave: Planificación regional y urbana, Latinoamérica, institutos

\begin{abstract}
Abstrac
In the context of the transformations of the geopolitical map after World War II, Latin America acquires a renewed interest for his continental neighbors. These international links are articulated from three areas of international relations: economical, technical and political cooperation, which will mark the pulse of transformations in the region in order to respond to the condition of "backwardness".

Understanding Latin America as "the" space for development planning, we propose a reading to advance in the shapes and mechanisms for the consolidation of the discipline in the region and its re-interpretations at local level, taking as subject matter the activity of two relevant centers: Centro de Estudios Urbanos y Regionales (CEUR) and Centro Interdisciplinario de Desarrollo Urbano y Regional (CIDU)
\end{abstract}

Key Words: Urban and regional planning, Latin America, institutions 


\section{PRESENTACIÓN}

En los años posteriores a la Segunda Guerra el escenario Latinoamericano se va modificando a partir de una renovación de sus vínculos continentales con los Estados Unidos que, con la reanudación de su política exterior, va a marcar el pulso de las transformaciones en el continente a partir del afianzamiento de un modelo de cooperación y financiación económica y técnica que permita dar respuestas a la condición de "atraso" y evite el creciente giro comunista de los intelectuales de izquierda latinoamericanos.

La renovación del glosario latinoamericano con la re-interpretación de la planificación en clave desarrollista y su caracterización como método de toma de decisiones tendiente al equilibrio territorial que incorpora factores políticos, sociales, económicos y también físicos, plantea la creación de nuevas teorías y enfoques, sumado a la configuración de instituciones de formación e investigación que puedan formar técnicos capaces de intervenir en el nuevo escenario latinoamericano, no sólo en el ámbito académico, sino principalmente para incorporarse al aparato burocrático del Estado en sus múltiples niveles.

En los últimos años se ha avanzado en la región en investigaciones orientadas a revisar las condiciones de inserción del planning de cuño norteamericano, esta serie de trabajos se articulan a partir de dos escalas y enfoques diferenciados: por un lado, la macro donde se revisan los "viajes de la planificación" desde la cultura urbana (Gorelik, 2013) y las transformaciones al interior de la disciplina (Almandoz, 2010) a escala regional, y en segundo término estudios centrados en casos particulares o redes académicas (Camacho, 2007; Jajamovich, 2015; Monti, 2015), advirtiendo la posibilidad de generar investigaciones comparativas que a partir de la articulación de estas dos dimensiones propongan nuevas interpretaciones sobre el período.

Nuestro trabajo se alinea en esta dirección, a partir del estudio de dos institutos claves en el cono sur como son el Centro de Estudios Urbanos y Regionales (CEUR-Argentina) y el Centro Interdisciplinario de Desarrollo Urbano y Regional (CIDU-Chile) con el fin de reconstruir los métodos y modelos de consolidación de las nuevas nociones de la planificación en clave cepalina, tanto en la Argentina como en Chile, y su traducción institucional e inserción universitaria.

Fernanda Beigel sostiene que la "existencia o no de tradiciones intelectuales nacionales y bases institucionales pre-existentes" (2010), se constituyen como elementos claves para identificar el éxito de inserción de ciertas ideas e instituciones en los complejos contextos nacionales. En este sentido, la Sociedad Interamericana de Planificación (SIAP-1956) se constituye como una institución promotora del proceso de institucionalización de la disciplina regional y urbana a escala regional, mientras que el desarrollo de los centros de investigación y formación nacionales, sumado a las sociedades profesionales constituyen los eslabones locales de estas redes de planificadores que en la década del sesenta va nutrir a la región a partir de las becas de formación en el exterior, los cruces académicos, la organización de seminarios de discusión y congresos, sumado a la definición de la agenda temática urbana a escala latinoamericana.

Partimos de la hipótesis de que el CEUR y el CIDU son los nodos nacionales respectivos de una red panamericana de la planificación, y que funcionaron como espacios de reproducción y posterior adaptación, revisión y creación de las teorías y modelos de la reformulada disciplina regional y urbana en la década del sesenta. En esta línea el trabajo pretende iluminar a través del relevamiento comparativo de los dos centros, las formas de institucionalización que adquirieron los mismos en función a las realidades locales particulares, sosteniendo que, a pesar de que compartían un ideario común y en muchos casos se retroalimentaban, sus características institucionales se presentan dispares.

A continuación de esta presentación, la segunda parte de la investigación busca identificar los límites implicancias de la incorporación de los conceptos de planificación y desarrollo en el glosario latinoamericano, analizando las líneas teóricas-metodológicas y los temas que van a guiar las indagaciones de los intelectuales latinoamericanos hasta principios de la década del setenta cuando se inicia el viraje a posiciones más críticas de base dependentistas.

La tercera y cuarta parte del escrito se orienta al reconocimiento de los modelos universitarios presentes en Argentina y Chile, reconociendo los ritmos internos de transformación con el fin de comprender las particularidades de inserción de los centros en los contextos nacionales sumado al estudio de las estructuras organizativas de los centros, sus objetivos de funcionamiento, sus vínculos con organismos estatales en sus múltiples niveles referidos a la asistencia técnica y los programas de formación, a partir del 
estudio de los documentos publicados, determinando las convergencias y divergencias de los centros a cada lado de la cordillera.

Por último se plantean las primeras aproximaciones comparativas de la investigación, avanzando en los vínculos entre los centros de investigación y su inserción en las estructuras académicas nacionales y la reinvención de los vínculos entre técnica y política.

\section{PLANIFICACIÓN Y DESARROLLO. LA RENOVACIÓN DEL GLOSARIO LATINOAMERICANO}

En los años posteriores a la Segunda Guerra Mundial, América Latina adquiere un renovado interés por sus vecinos continentales, en el marco de un escenario de transformación del mapa mundial que consolida a los Estados Unidos como potencia tanto en el campo político y económico pero también a nivel cultural y académico.

La amenaza comunista, con la caída del gobierno democrático guatemalteco incluida, es una de las preocupaciones de los círculos políticos estadounidenses. ${ }^{1}$ La Revolución Cubana (1959) abre un nuevo escenario para las relaciones inter-americanas. La radicalización cada vez más fuerte de grupos y personalidades latinoamericanas que asumen posiciones nacionalistas y anti-imperialista traslada la Guerra Fría al propio continente. ${ }^{2}$ Ante esta situación, Norteamérica potencia su política exterior con la denominada "Alianza para el Progreso" promovida por el presidente John Fitzgerald Kennedy ${ }^{3}$, iniciando un nuevo capítulo de la cooperación económica, política y cultural a escala regional que se propone para evitar el "mal ejemplo cubano", iniciando un período de fuerte promoción de programas de asistencia y cooperación financiera y técnica.

De esta manera, como sostiene Adrian Gorelik (2005), Latinoamérica se convierte en un territorio apto para experimentar hipótesis modernizadoras tendientes a afrontar los desequilibrios estructurales en la región a través de políticas que "buscaban localizar los obstáculos para el progreso económico a partir de una concepción que polarizaba sociedades desde su clasificación como tradicionales frente a sociedades que consideraba modernas" (Dos Santos, 2002; cit. Beigel, 2006). Esta concepción, se aleja de las etapas propuestas por Rostow identificando que "la problemática del desarrollo estaba ligada a la estructura económica y a la división internacional del trabajo" (Svampa \& Gras, 2015) y, por consiguiente, a la necesidad de abandono de un modelo productivo-exportador y su reemplazo por la industrialización de su economía. En este contexto, el desarrollismo surge como idea posibilitante para dar respuesta a los proceso de industrialización en las periferias, consolidando un debate que, con sus diferencias, va a estar presente en cada uno de los países latinoamericanos.

La planificación como noción que se incorpora al glosario latinoamericano en este período, es entendida como uno de los instrumentos de las políticas desarrollistas, que posibilita superar la condición de "atraso", en línea con el pensamiento de Harvey Perloff que la identifica como un método propositivo, como un programa político de organización y diseño de los sistemas económicos, territoriales y sociales, con un fuerte énfasis en la razón, la ciencia y la técnica en el marco de un sistema político sustentado en la participación y la democracia:

... un enfoque, un símbolo, y una actividad - que ha ayudado a poner en primer plano, y en la conciencia de los gobiernos y del público en general, la importancia y la conveniencia de tratar (operativamente) las relaciones entre las personas, los objetos físicos y las fuerzas ecológicas, de tratar de ver las cosas en su totalidad; de fijar metas tratando de averiguar la mejor manera de alcanzarlas; de tratar de coordinar e integrar las diferentes clases de mejoramiento físico y las actividades de desarrollo llevadas a cabo por el gobierno, con el objetivo de trabajar por un futuro mejor.... (Perloff, 1957)

\footnotetext{
1 Para ampliar sobre el tema ver el prólogo de Benedetta Calandra y Marina Franco (eds.), (2012) La guerra fría cultural en América Latina. Desafíos y límites para una nueva mirada de las relaciones interamericanas, Buenos Aires, Biblos.

2 El gobierno de Eisenhower, y luego los de Kennedy y Johnson, ensayaron distintas estrategias para desestabilizar a los revolucionarios cubanos: desde las sanciones económicas hasta llegar al bloqueo, las diplomáticas con la expulsión de Cuba de la OEA, las terroristas con el apoyo a los contrarrevolucionarios cubanos y las acciones encubiertas de la CIA para asesinar a Castro, y las militares fundamentalmente a partir de la invasión a Bahía de Cochinos, orquestada por la CIA.

3 La reunión se realiza en agosto de 1961 en la ciudad de Punta del Este, Uruguay, convocada por el Consejo Interamericano Económico y Social de la OEA.
} 
En definitiva es esta visión la que prevalece en el pensamiento latinoamericano, alentada por la política exterior norteamericana (Buena Vecindad 1933-1945 y posteriormente la Alianza para el Progreso 1961) a partir de la ampliación de organismos regionales de acción y la puesta en crisis de la hegemonía europea a nivel internacional, generando nuevos vínculos entre Estados Unidos y sus vecinos del sur.

Los intentos de inserción internacional de la región respondieron al concepto de desarrollo neocapitalista periférico, signado por una economía financiada por capitales externos y organizaciones internacionales (Fondo Monetario Internacional -1945 y ONU 1945), sumado a la acción de las elites políticas y el sector privado locales.

Simultáneamente se observa el aumento significativo de la cooperación internacional, siendo la Comisión Económica para América Latina y el Caribe (CEPAL-1948) el organismo emblemático en la definición del pensamiento económico orientado a:

\section{(...) facilitar la acción concertada para la reconstrucción económica (...) elevar el nivel de actividad económica (...) y mantener y reforzar las relaciones económicas de los países (...) tanto entre sí como con otros países del mundo (...) La comisión tiene la facultad de formular recomendaciones sobre cualquier asunto de su competencia, directamente a sus gobiernos miembros y a los organismos especializados. (Santa Cruz, 1984, p.125)}

La CEPAL se constituía como un organismo de referencia para la región, no sólo en términos económicos, sino también en la construcción de un cuerpo de ideas que guiarían el devenir del pensamiento latinoamericano. Autores como Marcos Kaplan advierten que la presencia de la planificación democrática en los postulados de la CEPAL era entendida como instrumento de crecimiento "que puede ser alcanzado respetando el sistema capitalista, el mercado libre y la empresa privada, y promoviendo la estabilidad social y la democracia política" (Kaplan, 1980).

Durante los primeros años la figura del argentino Raúl Prebisch ${ }^{4}$ (1948-1950), define el horizonte del organismo a partir de la propuesta de inserción internacional de las economías periféricas con el objetivo de evitar los desequilibrios por medio de la industrialización sustitutiva y el control de la inflación estructural y el desempleo. En los años cincuenta, se introducen innovaciones conceptuales que darán forma al repertorio teórico-conceptual de la CEPAL como las relaciones "centro-periferia", deterioro de los términos de intercambio, desequilibrio estructural de la balanza de pagos, inflación estructural, desempleo estructural, planificación del desarrollo en América Latina e integración regional. Este aparato conceptual que guió el pensamiento cepalino durante los años cincuenta y mediados de los sesentas presenta ciertas fisuras que, desde posiciones "externas" proponen una revisión del concepto de "centro y periferia" y la planificación como motor para el cambio. En esta línea, el economista francés François Perroux (1964), proponen una alternativa de la noción de implementación de la planificación en los países "en desarrollo" cuestionando la repetición del modelo keynesiano en aquellos cuyas economías dependían de variables externas, acuñando la "teoría de los polos de desarrollos" desde la cual intenta construir, a partir de la espacialización de las nociones económicas, un equilibro frente a la concentración territorial de las fuerzas económicas industriales.

Paralelamente y desde una perspectiva superadora de la enunciación conceptual, en los Estados Unidos se inicia un proceso de revisión de los principios de la planificación, que se caracterizó por el control de inversiones y la definición y programación de los recursos empleados en las diferentes actividades. Un exponente de esta "nueva" visión es el planificador urbano John Friedmann que enfatiza la complejidad del concepto de la planificación, entendiendo que la misma puede considerarse simplemente como la acción de la razón sobre una red de actividades en movimiento, a través de la intervención de ciertas estructuras y procesos de decisión (Faludi, 1974). Sostenemos que abordar la planificación como programación de la relación entre los fines y los medios desde modelos conceptuales sustentados en el análisis cuantitativo y numérico, donde la definición de objetivos y la identificación de las variables para el desarrollo de políticas y métodos de evaluación constituyen las bases de una disciplina renovada en la que el territorio se transforma en soporte de las actividades y, por ende, se produce un borramiento de la condición física en pos de un conjunto de relaciones que involucra la economía, la sociedad, la política y también el territorio.

\footnotetext{
4 Raúl Prebisch fue uno de los fundadores del Banco Central Argentino en 1935. Entre 1950 y 1963 fue Secretario Ejecutivo de la Comisión Económica de las Naciones Unidas para América Latina y el Caribe (CEPAL) y posteriormente Secretario General de la Conferencia de las Naciones Unidas sobre Comercio y Desarrollo (UNCTAD). Entre octubre de 1955 y enero de 1956 elaboró, para el gobierno de facto de la Revolución Libertadora, un diagnóstico de la situación económica de Argentina y un plan de acción para resolver los problemas económicos.
} 
En Latinoamérica, la preeminencia del pensamiento cepalino en la década del cincuenta y sesenta sustentado en la construcción de un conjunto de contenidos básicos y de teorías y modelos, se orienta a explicar la condición de desarrollo/subdesarrollo y la polaridad centro/periferia. Se reconocen tres conceptos claves explicativos de la condición de las economías periféricas: la heterogeneidad estructural, la especialización productiva y el desarrollo desigual centrado en el par centro-periferia. De esta forma, la planificación democrática se constituye como un instrumento clave para el desarrollo en el continente, no sólo en términos de ideas y teorías, sino también a partir de organismos panamericanos y programas de asesoramiento políticos, de intervención económica e intercambio de las élites intelectuales, que consolidan una mirada que entiende a la planificación como motor de la transformación.

En nuestra disciplina estas ideas de la planificación como método de toma de decisiones, cuyo objetivo es el tan ansiado equilibrio territorial, va a renovar el repertorio teórico-metodológico abandonando paulatinamente posicionamientos centrados en la forma física por posiciones más abstractas que entrecruzan variables económicas, políticas, sociales y territoriales para definir los lineamientos de actuación. Entendiendo al territorio como soporte, la perspectiva regional adquiere un papel significativo dentro de las teorías y prácticas disciplinares en parte para dar respuesta a la polarización y los desequilibrios coyunturales que determinaron el desarrollo inequitativo de los países latinoamericanos. Reforma agraria y la reforma urbana completan los temas centrales de la disciplina en el período entendiendo, como afirma Hauser, a "la ciudad como motor de cambio del desarrollo económico y por ende de un amplio proceso social" (1967) y, por lo tanto, la planificación de una ciudad no puede limitarse a la simple estructuras de las áreas urbanas, sino que comprende además la necesidad de restaurar y organizar el medio como lugar agradable para vivir y trabajar asumiendo la planificación un carácter integral que debe hacer frente a la necesidad de coordinar la planificación física con la económica, social y administrativa. Esta idea debatida en los círculos de intelectuales deja implícita la interdisciplina como método de actuación, abandonando el rol del profesional independiente con un fuerte anclaje en las forma física, por el trabajo en equipo, donde la conjunción de diferentes disciplinas y metodologías redefinen los abordajes y consolidan aproximaciones más abstractas basadas en métodos científicos de recolección de datos, donde la variable física es una más junto con la economía, lo político y lo social.

\section{UNIVERSIDAD E INVESTIGACIÓN. MODELOS DE ACCIÓN.}

Entre 1955 y 1973, el dominio de la ciencia y de la técnica se convierten en los pilares de los modelos universitarios en el continente desempeñando un rol estratégico en el desenvolvimiento económico de los Estados. La productividad intelectual y científica es fomentada desde las universidades, los centros de investigación y los nuevos organismos estatales como el Consejo Nacional de Investigaciones Científicas y Técnicas (CONICET-1958) en Argentina o el Comisión Nacional de Investigación Científica y Tecnológica (CONICYT-1967) en Chile que, que a través de becas y subsidios dinaminazon la formación de posgrado en el extranjero y subsidiaron el desarrollo de proyectos de innovación teórica y técnica.

El fortalecimiento científico en cada país se encuentra en estrecha relación a la necesidad de formar recursos humanos acordes al modelos económico del desarrollo. Esta situación plantea el reemplazo de un modelo educativo centrado en el ejercicio de la profesión por la consolidación de una estructura universitaria cientificista, con un fuerte rol social sustentado en una concepción universalista de la cultura, donde investigación y asistencia técnica se convierten en los pilares del cambio.

Este proceso no se presenta ajeno a la modernización de las ciencias sociales a escala internacional (principalmente sociología, historia, economía y desarrollo urbano), reconociendo una creciente centralidad de estas nuevas temáticas en las agendas de gobierno y, por lo tanto, el aumento de los recursos para la financiación de proyectos de investigación y centros de formación.

$\mathrm{Si}$ bien estos principios se mantienen en los dos países, los mecanismos, modelos y condiciones tanto académicos como políticos van a marcar el devenir disciplinar, consolidando formas de organización diferenciadas a cada lado de la cordillera.

Si en Argentina la renovación y modernización de las universidades y su viraje a una concepción centrada en la investigación, la ciencia y la técnica va a caracterizar a la denominada "década de oro de las universidades", entre 1955 y $1966^{5}$, los años posteriores al golpe cívico militar y la respectiva intervención de las universidades y su pérdida de autonomía y autarquía van a cristalizar el desplazamiento y

5 Este periodo se inicia con la renovación de la universidad posterior al golpe cívico militar del año 1955, en la búsqueda por la diferenciación del modelo universitario peronista. 
concentración "del capital académico a los centros privados que alojaban a los universitarios expulsados del ámbito estatal"(Beigel, 2006) incrementando la financiación externa que, en los ámbitos universitarios había adquirido un fuerte rechazo debido a la consideración de subordinación y dependencia de la universidad al "imperialismo" norteamericano personificado por la acción de organismos supranacionales como la ONU, o el BID o bien, la acción filantrópica de la Fundación Ford.

En este escenario de conflictividad política, los vínculos entre universidad y producción científica se transforman, adquiriendo los centros e instituciones privadas una creciente centralidad que les permite administrar sus fondos de manera independiente. Esta condición sin embargo, no implica el cambio del paradigma desarrollista-cientificista, lo que permite trazar ciertas continuidades del proceso de institucionalización y consolidación de la disciplina anterior al golpe de Estado de 1966, pero que va a introducir transformaciones en línea con el giro dependentista en la región que se inicia en los primeros años de la década del setenta.

En Chile, la situación política-institucional entre mediados de la década del cincuenta y 1973 presenta condiciones diferenciadas de la Argentina, caracterizadas principalmente por la estabilidad política, la expansión del sistema universitario y su papel como espacio de confluencia de actores extranjeros, organismos y fuentes de financiación externa que permite consolidar su liderazgo como espacio de regionalización de las ciencias sociales.

La continuidad de gobiernos progresistas entre 1964 y $1973^{6}$ y el incremento de financiación estatal a las universidades, posibilitó concentrar la investigación científica en el sistema universitario sumado a la generación de centros privados o centros regionales dependientes de organismos internacionales que, en menor medida, van a terminar de definir un entramado institucional combinado vinculado a las estructuras universitarias a través de convenios de asistencia técnica.

La fragilidad política-institucional de la región ${ }^{7}$ en este periodo se contrasta con la realidad chilena, convirtiendo a este país en un espacio de confluencia de cientistas sociales del todo el mundo, en un "espacio de internacionalización y plataforma de regionalización" (Beigel, 2010), que respondía al modelo desarrollista cepalino y que contaba con el apoyo político, económico y teórico/técnico del reformismo norteamericano. Esta situación dinamiza el medio chileno identificando un aumento sustancial de la productividad académica, donde los cruces entre actores de la región y del medio internacional van a consolidar teorías y modelos para dar solución a la condición del "sub-desarrollo" desde la propia región y, por lo tanto, iniciar un periodo de revisión de las teorías y metodologías que pretenden actuar en los procesos de transformación.

La continuidad de las políticas universitarias fue consolidando en la región un perfil cada vez más radicalizado y transformado, donde los intelectuales latinoamericanos van a ir extremando sus posiciones frente al modelo norteamericano, convirtiendo al Chile en "un estandarte del socialismo democrático y líder tercermundista" (Beigel, 2010).

El aumento de la oferta académica a nivel de formación de posgrado, sumado a la consolidación de centros e institutos que contaban con financiación mixta público/privada - nacional/internacional hicieron que Chile y, particularmente Santiago, actuara como un faro a nivel regional sumado a consolidarse como un espacio de renovación de las teorías y conceptos que permitieron una renovación del glosario desarrollista en clave dependentista pensado desde y para Latinoamérica.

El golpe de Estado de 1973 del general Augusto Pinochet, clausura el circuito de crecimiento y expansión de las ciencias sociales de la región, en un clima político convulsionado que va a ir acrecentando sus rasgos de violencia institucional. Si Argentina se convirtió por tres años (1973 y 1976) en uno de los primeros posibles destinos de los actores chilenos para escapar del gobierno de Pinochet a través de las redes que se habían consolidado en los años anteriores y, particularmente a través del Programa de emergencia de CLACSO para refugiados chilenos, el Golpe cívico militar más violento de la historia Argentina, comandado por el General Videla en el año 1976, va a clausurar el modelo de investigación en el país y consolidar el desplazamiento hacia México que intensifica una segunda ola migratoria producto del exilio de académicos en la región.

6 Las presidencias del período son: 1952/1958 Carlos Ibañez del Campo; 1958/1964 Jorge Alessandri Rodríguez; $1964 / 1970$ Eduardo Frei Montalva; 1970/1973 Salvador Allende

7 El triunfo del candidato demócrata cristiano en Brasil en el año 1964 y el posterior golpe de Estado, inician un período de inestabilidad política-institucional en la región. 


\section{UN INSTITUTO A CADA LADO DE LA CORDILLERA: EL CEUR Y EL CIDU COMO MODELOS DIFERECIADOS DEL ACCIONAR DE LOS CENTROS}

El estudio de los centros referidos a la disciplina urbana en cada país se presenta como una oportunidad para revisar las formas de internacionalización de la disciplina urbana en el contexto de la circulación internacional de las ideas, sumado a la discusión sobre la dependencia académica que permite una revisión de los modos en que ciertas ideas son transportadas y adaptadas en ámbitos diferentes al de su producción y que, en ocasiones, permiten discutir el tradicional par polar centro-periferia en su sentido más amplio.

En el año 1961 la Misión Técnica de SIAP encabezada por Francis Violich publica el informe "La enseñanza de la planificación en América Latina" en el cual se establecen los lineamientos para la conformación de centros e institutos de investigación y formación en la región acorde a los requerimientos de la disciplina en la región.

El programa se propone sobre la base integral que "debe reconocer tres niveles a los cuales el proceso del planeamiento debe ser aplicado: nacional, regional y local/urbano; y debe incluir cursos orientados al planeamiento para el desarrollo nacional, regional y urbano, sumado a actividades de investigación, programas de extensión y educación pública y asistencia técnica" (SIAP \& Ford Foundation, 1961).

En esta línea, tanto el CEUR como el CIDU se presentan como exponentes de los diferentes procesos de expansión institucional en cada país. Mientras que los orígenes del Centro de Estudios Urbanos y Regionales (CEUR) se insertan en el sistema púbico universitario entre 1962 y $1966^{8}$, su posterior desarrollo está vinculado al recientemente creado Instituto Torcuato Di Tella (ITDT-1958), confirmando la centralidad que adquieren los centros privados como espacios para el desarrollo científico-técnico en el marco de las políticas intervencionistas universitaria del gobierno de facto de Juan Carlos Onganía. Esta condición es a su vez entendida por el ITDT a partir de la ampliación de su estructura institucional argumentando que "la tarea del instituto está centrada en la modernización cultural del país, con la esperanza de contribuir así a desatar el nudo cultural que traba nuestro desarrollo"(ITDT, 1966)

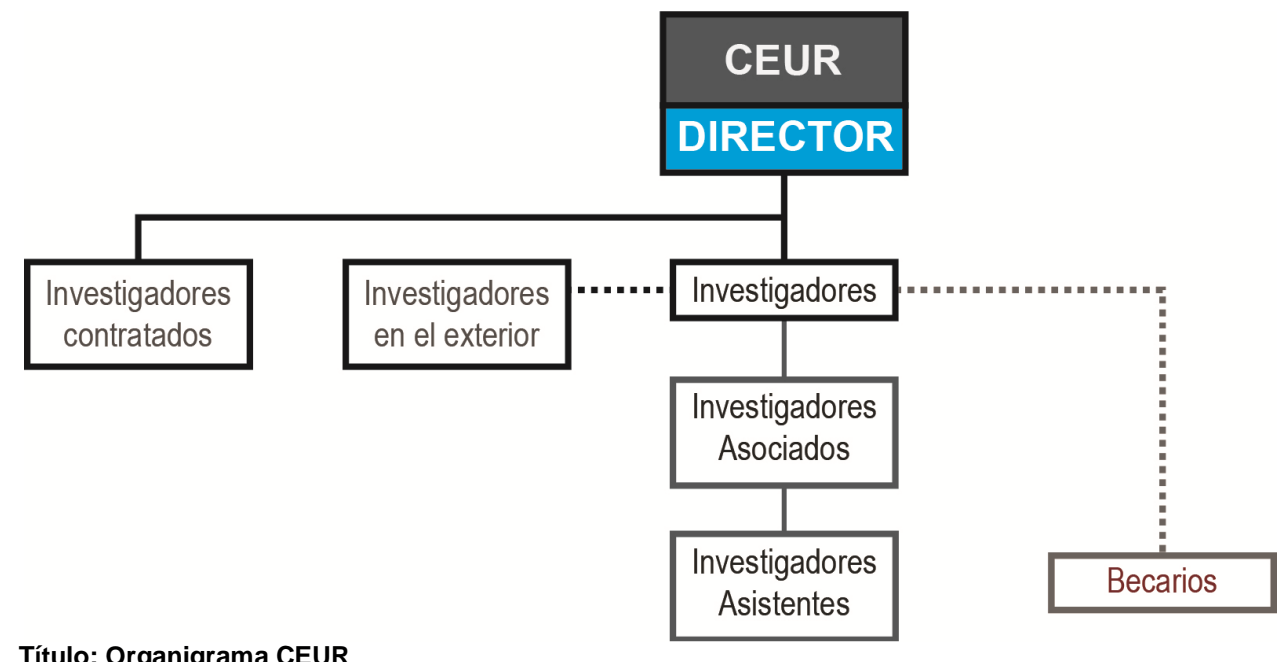

Título: Organigrama CEUR

Elaboración propia a partir de datos del ITDT

El Centro Interdisciplinario de Desarrollo Urbano y Regional (CIDU) surge en el año 1966 como iniciativa de un conjunto de docentes y actores de la Pontificia Universidad Católica de Chile, con el fin de dar respuestas a la necesidad de generar un programa de enseñanza e investigación del proceso de desarrollo urbano. La dependencia al Rectorado de la universidad marca su "autonomía" frente al accionar de las unidades académicas (las facultades), potenciando el desarrollo de la investigación desde las estructuras universitarias, pero a través de centros especializados que permitan articular e independizar la formación de grado del posgrado.

8 La trayectoria del CEUR se inicia en la ciudad de Rosario en el año 1962 con la conformación del Instituto Regional y Urbano del Litoral (IPRUL) y, posteriormente, se produce la mudanza a la Universidad de Buenos Aires como Equipo de Estudios Urbanos y Regionales (EEUR). En 1966 y tras los hechos de la denominada "Noche de los bastones largos" que del gobierno de facto de Juan Carlos Onganía, el CEUR abandona la inserción en el ámbito público para insertarse en el ITDT como centro asociado. 
La condición de centro "privado" del CEUR se refleja en su estructura institucional. En este sentido los centros del ITDT dependen del Director del Instituto que es elegido por el Consejo de Administración. El organigrama del CEUR lo encabeza un Director, seguido por el grupo de investigadores contratado full time, los grupos de investigadores asociados y asistentes respectivamente sumado al grupo de becarios. La dirección del mismo es bianual y se define a través de un acuerdo interno identificando que entre 1966 y 1974 el cargo es ocupado por miembros del grupo de investigadores (Jorge Enrique Hardoy 1966/70; Alejandro Rofman 1970/71 y Oscar Yujnovsky 1972/74).

La dependencia al Rectorado del CIDU representa una estructura institucional más compleja donde se articulan espacios de representación tanto a nivel de docencia con el Consejo de Profesionales como en la estructura mixta del Comité Directivo conformado por miembros de la Universidad y representantes de organismos estatales vinculados al desarrollo regional y urbano. El organigrama del centro está encabezado por un director, seguido del subdirector y acompañado por un coordinador de docencia, un jefe del curso de egresados y un jefe de proyecto de investigación y asistencia técnica. En los primeros años de funcionamiento el cargo de director es ejercido por Ricardo Jordán, seguido por Guillermo Geisse.

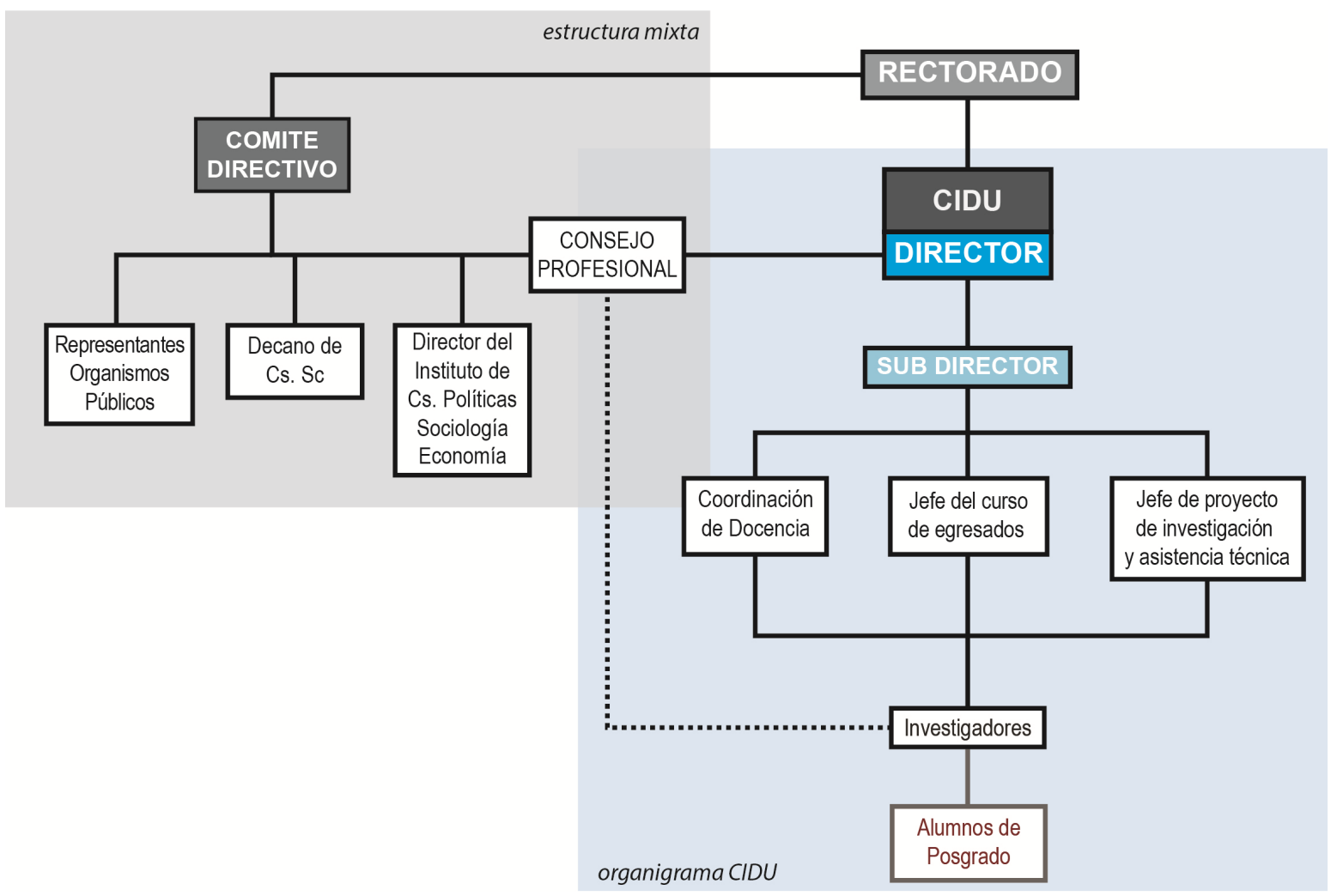

Título: Organigrama CIDU

Elaboración propia a partir de datos del Instituto

Si bien las formas de financiamiento de los dos centros presentan características diferenciadas, resulta posible establecer ciertas condiciones análogas que permiten una re-lectura de la asistencia financiera externa. En primer lugar la incorporación de la asistencia técnica como objetivo de funcionamiento, presenta una característica que permite traspasar el ámbito académico y consolidar el rol de los expertos como actores centrales de la diagramación de la agenda de la región a partir de la realización de proyectos e investigaciones que vinculen la actividad académica con el medio, ya sea para organismos públicos nacionales como internacionales o bien, la actividad filantrópica de fundaciones como Ford o Rockeffeller que van a inyectar recursos a los centros de la región a través de la firma de convenios específicos que abarcan desde trabajos de investigación y seguimiento, formación de personal, diagramación de oficinas públicas o bien el intercambio de docentes y becarios a escala internacional. Un punto distintivo de los mecanismos de financiación de los centros se encuentra vinculado a la actividad de los investigadores, mientras que en el CEUR los fondos provienen de una combinación entre el ITDT y el CONICET, e en el 
CIDU se enmarcan dentro del sistema de contratación de la universidad, conformando dos modelos distintivos vinculados estrechamente a las estructuras institucionales que le dan forma.

Los diferentes esquemas de enseñanza propuestos por estos centros son deudores de las propuestas de la Misión Técnica de SIAP, identificando la centralidad de este tema en el funcionamiento de los institutos con el fin último de "preparar profesionales capacitados para actuar eficientemente en el campo de la planificación para el desarrollo urbano-regional y en la promoción de las actividades necesarias para alcanzar una más cabal comprensión del fenómeno de urbanización, como base para la formulación de políticas y programas de desarrollo urbano-regional" (CIDU, 1968).

En el CEUR, la estructura de los programas de enseñanza va a sufrir modificaciones en el tiempo. Deudor de la experiencia del IPRUL ${ }^{9}$, mucho más cercana a los principios de SIAP, en los primeros años del CEUR en el contexto institucional del ITDT la propuesta de formación estaba orientada a la conformación de un programa de docencia tendiente a lograr un "mayor perfeccionamiento teórico y metodológico" (ITDT, 1967), destinado a los investigadores asistentes y al grupo de becarios del centro.

En paralelo, el CIDU empieza a desarrollar su programa de formación. identificando dos escalas de aproximación diferenciada: la nacional-metropolitano y la escala comunal. Esta división supone identificar problemas y abordajes diferenciados, sumado a la identificación de actores e instituciones que, en cada uno de los niveles, van a involucrase en el proceso de la planificación regional y urbana en línea con los conceptos desarrollistas.

En el marco de la estructura universitaria, la propuesta realizada por el CIDU se organiza en tres niveles, el primero vinculado a la enseñanza de pre-grado ${ }^{10}$, donde se plantean una serie de cursos introductorios orientados a las carreras de arquitectura, economía, derecho, ingeniería y sociología.

El segundo nivel corresponde a los cursos básico, orientados a los egresados de las carreras afines. Los mismos se orientan a la consolidación de una mirada coherente de los procesos y problemas de la urbanización en términos teóricos, técnicos y metodológicos, desde abordajes cuantitativos o cualitativos dependiendo de la escala de actuación y los temas de estudio.

Por último, los cursos de especialización surgen de la articulación entre la" experiencia de los alumnos, los cursos básicos y las expectativas de ocupación" (CIDU, 1968, 26), de esta forma se proponen una serie de créditos finales (40) divididos en tres trimestres, que se logran a partir de la aprobación de cursos obligatorios y electivos, sumado a la realización de una tesis o trabajo final referido a un campo de investigación específico.

Las áreas de estudio sugeridas por el CIDU son vivienda y equipamiento comunitario, desarrollo social, administración del desarrollo urbano-regional, economía de la empresa política y transporte urbano. Una de las condiciones requeridas para la realización de la especialización refiera a la dedicación exclusiva en sus actividades, situación que plantea la necesidad de financiación a través de un programa de becas mixtas entre el CIDU y el sector público como el "Ministerio de Vivienda y Urbanismo (MINVU), la Corporación de Fomento de la Producción (CORFO) y la Oficina de Planificación Nacional (ODEPLAN).

A partir de 1968 el CEUR y el CIDU, en el marco de la creación de Consejo Latinoamericano de Ciencias Sociales (CLACSO), presentan a Naciones Unidas la propuesta del "Programa Latinoamericano de Investigación y Docencia en el campo de la urbanización", en un esfuerzo compartido donde se dictaban cursos y seminarios en cada sede, pero que contaba con el intercambio de becarios y docentes que retroalimentaban las dos experiencias en paralelo.

En el año 1972 el CEUR comienza a delinear el "Programa de Formación de Investigadores en Desarrollo Urbano y Regional"11, en el marco de lo planteado por la Comisión de Desarrollo Urbano y Regional de CLACSO contando a su vez con el auspicio del ILPES. La propuesta tendiente a resolver el déficit de formación de investigadores en el área de la planificación, reconociendo la especificidad de la realidad Argentina y latinoamericana se articula a partir de una concepción dinámica de interacción entre "docentesestudiantes-graduados" en un programa sustentado en la investigación y la formación a través del desarrollo de seminarios y cursos de lecturas. La centralidad otorgada a la teoría y a la crítica constituyen la matriz del

9 Para ampliar sobre la trayectoria de la formación de posgrado en el IPRUL ver Monti, A. (2013) Redes, instituciones y planificación. El caso del Instituto de Planeamiento Regional y Urbanos del Litoral. 1955-1965. Tesis para optar por el grado de Magister. Maestría en Historia y Cultura de la Arquitectura y la Ciudad. Universidad Torcuato Di Tella. Buenos Aires

10 El nivel universitario en Chile se denomina pre-grado

11 Para ampliar sobre el Programa ver CLACSO (1973). Bases para un programa Latinoamericano de postgrado en Ciencias Sociales. Buenos Aires, CLACSO, p. 39 
programa, orientado "a la ampliación y enriquecimiento del plan general de investigación del CEUR" (ITDT, 1972), abandonando la asistencia técnica y la formación de "profesionales", para dar paso a la formación de investigadores y teóricos, cada vez desde abordajes más alejados de la forma física.

En el CIDU, se mantenía la estructura de años anteriores, equiparando los trabajos de asistencia técnica con la investigación, condición que permite identificar un perfil de formación que entiende al planificador como "un profesional orientado a la acción y, más específicamente, ésta debe darse dentro del marco decisionario de la estructura social de los países latinoamericanos" (CLACSO, 1973).

\section{NORTE - SUR / SUR - SUR}

Si bien esta investigación se encuentra en proceso de avance resulta posible resaltar algunas aproximaciones parciales que permiten re-pensar los vínculos entre técnica y política a escala regional y local.

En primer lugar la renovación del glosario latinoamericano de la planificación se presenta en un momento particular del debate político a escala regional. La presencia de los Estados Unidos a través de múltiples mecanismos de cooperación económica, política pero también técnica permite redefinir los vínculos norte sur, desde una posición que intenta alejarse de la noción de "influencia" para dar paso a una comprensión más compleja basada en el concepto de re-apropiación y re-definición que pone en crisis un polo productor y otro receptor sin transformación. En este sentido los alcances, principios y enfoques de la planificación en la región van a ir transformándose en el tiempo desde posiciones iníciales más vinculadas a una condición cercana al posicionamiento etapista de Rostow, para transformarse muy tempranamente desde un mirada que identifica las condiciones estructurales históricas de la región y, por consiguiente, la necesidad de innovación en el diseño de medidas tendientes al desarrollo y el equilibrio territorial, para pasar en los últimos años de la década del sesenta a posicionamientos más críticos vinculados a la teoría de la dependencia que tiene su origen en la propia región.

En línea con el planteo de Gorelik (2005), la planificación como método va presentar una temporalidad diferenciada de los otros conceptos utilizados en la región: modernización y el desarrollo, planteando una condición que entiende a la ciencia y la técnica desde la búsqueda del bien común y, por lo tanto, desproblematizada del posicionamiento ideológico, identificando el hacer experto desde una lectura que asume su intervención independizada de los vaivenes de la política y de las críticas de la propia disciplina, asumiendo el valor metodológico de la planificación como método por sobre el ¿qué? y el ¿cómo? o ¿desde donde? se establecen los lineamientos. La concepción de la planificación como método, como un programa técnico, sigue manteniendo su vigencia tanto en el contexto del desarrollismo como de la crítica dependentista, condición que va a tener su quiebre definitivo a partir de la consolidación de los gobiernos de facto en la región y la crisis del sistema capitalista producto de la denominada "crisis del petróleo".

El afianzamiento a escala latinoamericana y nacional de centros e institutos posibilitó, sumado al fortalecimiento de los vínculos académicos norte-sur, sur-sur, la consolidación de espacios de debate y condensación de temas y paradigmas referidos a las implicancias del desarrollo en estas latitudes, donde los centros juegan un rol central como nodos locales de una red latinoamericana de planificadores regionales y urbanos. En este sentido tanto el CEUR como el CIDU son exponentes de esta condición, identificando que en la simultaneidad de su creación y las similitudes de sus enfoques teóricos existen condiciones análogas vinculadas a procesos de institucionalización y regionalización de la disciplina a escala Latinoamericana, donde SIAP y CLACSO actúan como asociaciones regionales aglutinadoras de las experiencias locales.

Pero a pesar de las "deudas" comunes, también cada centro a uno y otro lado de la cordillera presentan características diferenciadas referidas a los modelos con que cada país consolidó sus vínculos entre ciencia y universidad. Mientras que en Argentina el desarrollo de la investigación post 66 se radico por fuera de las estructuras de las universidades públicas tradicionales, en Chile fueron las casas de altos estudios las que concentraron las producciones científicas, situación que marca caminos diferenciados que van a tener un recorrido signado por la inestabilidad política e institucional que caracterizo a la región, donde los sucesivos golpes de Estado no sólo van a impactar directamente en las estructuras universitarias, sino que también, condenan a los investigadores al exilio y interrupción definitiva de un modelo de investigación y asistencia técnica que se había consolidado, con sus diferencias y vaivenes, en la región. 


\section{BIBLIOGRAFIA}

ALMANDOZ, A. (2010). From urban to regional planning in Latin America, 1920-50, Perspectives Vol. 25, №. 1, enero.

(2006), Urban planning and historiography in Latin America, Progress in planning (65)

BEIGEL, F. (2006). Vida, muerte y resurrección de las "teorías de la dependencia". En Crítica y teoría en el pensamiento social latinoamericano (pp. 287-326). Buenos Aires: CLACSO.

---------- (2010). Autonomía y dependencia académica: Universidad e investigación científica en un circuito periférico : Chile y Argentina (1950-1980). Buenos Aires: Editorial Biblos.

CALANDRA, B., \& FRANCO, M. (2012). La guerra fría cultural en América Latina: desafíos y límites para una nueva mirada de las relaciones interamericanas. Buenos Aires: Biblos.

CAMACHO, L. (2007). Sociedad Interamericana de Planificación, SIAP 50 años Vida institucional y programática, Revista Bitácora Urbano Territorial, enero-diciembre.

CIDU. (1968). EI CIDU, Teoría y práctica de un programa de docencia e investigación en el campo de la urbanización. Cuadernos de desarrollo urbano y regional, (7).

CLACSO. (1973). Bases para un programa latinoamericano de postgrado en Ciencias Sociales.

FALUDI, A. (1974). A reader in planning theory. Oxford [etc.: Pergamon Press.

GORELIK, A. (2005). La producción de la "ciudad latinoamericana". Tempo Social. Revista de Sociología da USP, 17(1), 111-133.

-(2014). Miradas cruzadas. El viaje latinoamericano del planning norteamericano, Bifurcaciones, (18), septiembre/noviembre. Disponible en. http://www.bifurcaciones.cl/2014/12/gorelik/

HAUSER, P. (Ed.). (1967). La urbanización en América Latina: documentos del Seminario sobre Problemas de Urbanización en América Latina. Buenos Aires: Solar/Hachette.

ITDT. (1967). Memoria y balance. 1965/1966. ITDT.

JAJAMOVICH, G. (2013). Itinerarios de la Comisión de Desarrollo urbano y regional de CLACSO entre 1967 y 1973: entre la técnica y la política, las ciencias sociales y la planificación urbana y regional. Ponencia presentada en el Congreso XXIX ALAS, Santiago de Chile.

KAPLAN, M. (1980). Sociedad, política y planificación en América Latina. México: Universidad Nacional Autónoma de México, Centro de Estudios sobre la Universidad.

MONTI, A. (2013). Redes, instituciones y planificación. El caso del Instituto de Planeamiento Regional y Urbanos del Litoral. 1955-1965 (Tesis de Maestría). Universidad Torcuato Di Tella, Buenos Aires.

PERLOFF, H. S. (1957). Education for planning: city, state [and] regional. Baltimore, Md.: John Hopkins.

PERROUX, F. (1964). La economía del siglo XX. Barcelona: Ariel.

Santa Cruz, H. (1984). Cooperar o perecer: el dilema de la comunidad mundial. 1, 1,. Buenos Aires: Grupo Editor Latinoamericano.

SIAP, \& FORD FOUNDATION. (1961). La enseñanza de la planificación en la América Latina. San Juan, P.R.: La Sociedad.

SVAMPA, M., \& GRAS, C. (2015). El desarrollo en disputa: actores, conflictos y modelos de desarrollo en la Argentina contemporánea. 